\title{
Material Fragmentation as Dissipative Process of Micro Rotation Sequence Formation: Hybrid Model of Excitable Cellular Automata
}

\author{
D. D. Moiseenko ${ }^{1, \text { a) }}$, V. E. Panin ${ }^{1,2}$, P. V. Maksimov ${ }^{1}$, \\ S. V. Panin ${ }^{1,2}$, and F. Berto ${ }^{3}$ \\ ${ }^{1}$ Institute of Strength Physics and Materials Science SB RAS, Tomsk, 634055, Russia \\ ${ }^{2}$ National Research Tomsk Polytechnic University, Tomsk, 634050, Russia \\ ${ }^{3}$ University of Padova, Vicenca, 36100, Italy \\ a) Corresponding author: mdd@ispms.tsc.ru
}

\begin{abstract}
The authors have developed a multi-level model of energy propagation along interfaces between the various structural elements of a solid with taking into account mutual energy transformations of various kinds. They have also designed a computer simulation tool based on the excitable cellular automaton (ECA) method. An algorithm for calculating the local moments of forces has been developed for the case of material rotation and torsion. The relationship for the accumulated elastic energy is supplemented with a dissipation term. Numerical experiments have been carried out on high-energy impact on polycrystalline copper specimens with different grain sizes. The paper shows that during the nanostructuring of material surface layer, the dissipation of elastic energy gives rise to the rotation of structural elements. This makes it possible to prevent the occurrence of stress concentrators with peak values typical of coarse-grained specimens and reducing their mechanical properties.
\end{abstract}

\section{INTRODUCTION}

The governing statement of physical mesomechanics is that it treats any solid as a hierarchically organized system that contains a 3D crystalline subsystem and the array of all the surfaces and interfaces, which are independent 2D subsystems. Within these planar subsystems, primary rotational and wave flows of mass and energy are propagated under deformation. While these flows develop, defect structures emitted from the boundaries (interface) into a 3D crystal emerge in the planar subsystems. A new discipline, physical mesomechanics of materials, is successfully developing this concept. [1]. The analytical theory that describes the processes of curvature formation in rotational and wave flow of defects is formulated in [2].

However, there are practically no models available that would adequately describe the occurrence of rotational modes of deformation along the interfaces in a loaded solid. Dislocation-based approaches are normally used to describe the behavior of a bulk crystal and diffusion theories, to describe the mass transfer along the interfaces. In this context, it is of great current importance to design models that explicitly take into account the local moments of forces as well as the related rotational component of the plastic flow of the material.

We have developed a mathematical model based on the thesis that deformation is caused by the redistribution of energy between various structural elements of a solid and transformation of its various parts into each other. We also designed a computer simulation tool based on excitable cellular automaton method. A three-dimensional model of designing the grain structure of polycrystalline materials was constructed with explicit consideration of the crystal lattice orientation for each grain. An algorithm for calculating the local moments of forces has been developed for the case of material rotation and torsion by explicit calculation of angular velocities in the mass transfer flow. The relation for the accumulated elastic energy was supplemented with a dissipative term.

International Conference on Physical Mesomechanics of Multilevel Systems 2014

AIP Conf. Proc. 1623, 427-430 (2014); doi: 10.1063/1.4898973

(C) 2014 AIP Publishing LLC 978-0-7354-1260-6/\$30.00 


\section{BASICS OF EXCITABLE CELLULAR AUTOMATON APPROACH}

The excitable cellular automaton method involves simulating the distribution of mechanical energy flows in a specimen subjected to external mechanical impact (tension, compression, etc.). The specimen being simulated is represented as a cellular automaton, which is a network of interacting active elements. The network is divided into clusters each responsible for a separate grain with its own orientation of the crystalline lattice characterized by the Euler angles $\varphi, \psi, \eta$. The input parameters of the model are the initial values of the stress vectors at the centers of the elements $\left\{\mathbf{f}_{i}^{0}, 0 \leq i \leq I-1\right\}$, where $I$ is the total number of elements. The offered calculation algorithm is implemented for both FCC and simple cubic packages of active elements. This is necessary in order to avoid contradiction between the imposed "model" symmetry and that of the crystalline structure of the material under simulation.

At every $n$-th time step of the algorithm, the following operations are implemented. The stress $\boldsymbol{\sigma}_{i k}^{n-1}$ at the interface between $i$-th element and its neighboring $k$-th one $(1 \leq k \leq K, K$ is the total number of neighbors) is calculated as the resulting projection of the stress vectors of these elements onto normal to their boundary, the normal being directed from this neighbor towards the $i$-th element (Fig. 1(a)):

$$
\boldsymbol{\sigma}_{i k}^{n-1}=\mathbf{n}_{i k}\left(\mathbf{f}_{i}^{n-1}, \mathbf{n}_{i k}\right)+\mathbf{n}_{i k}\left(\mathbf{f}_{k}^{n-1}, \mathbf{n}_{i k}\right) .
$$

In the offered algorithm, the elements located at the first coordination sphere of an element are regarded as neighbors of this element. The flow rate of a substance through the considered interface $\mathbf{v}_{i k}^{n-1}$ under the stress $\boldsymbol{\sigma}_{i k}^{n-1}$ is calculated by Turnbull formula:

$$
\mathbf{v}_{i k}^{n-1}=m_{i k} \cdot \boldsymbol{\sigma}_{i k}^{n-1}
$$

Here $m_{i k}$ is the "mobility" of the material at the interface between the $i$-th element and its neighboring $k$-th element:

$$
m_{i k}=\frac{c}{\sqrt{Y_{i} Y_{k}}} e^{-\frac{\left(Y_{i}-Y_{k}\right)^{2}}{Y_{i} Y_{k}}} e^{-\frac{Q_{i k}}{k_{B} T_{i k}}},
$$

where $Y_{i}, Y_{k}$ are Young's modulus values of the $i$-th element and its $k$-th neighbor, $c$ is effective velocity of a medium response to external mechanical impact; $k_{\mathrm{B}}$ is Boltzmann constant; $Q_{i k}$ is energy of the interface between these elements at the $(n-1)$-th time step:

$$
Q_{i k}=\gamma_{\mathrm{HAGB}} \frac{\left|\theta_{k}-\theta_{i}\right|}{\theta_{\mathrm{HAGB}}}\left(1-\ln \frac{\left|\theta_{k}-\theta_{i}\right|}{\theta_{\mathrm{HAGB}}}\right),
$$

where $\gamma_{\mathrm{HAGB}}$ is the maximum boundary energy corresponding to the maximum angle of crystalline lattice misorientation, $\theta_{\mathrm{HAGB}}$ is maximum angle of misorientation, $\theta_{i}, \theta_{k}$ are effective lattice orientation angles of the $i$-th element and its $k$-th neighbor; $T_{i k}$ is the value of temperature for the considered interface.

Angular velocities of the rotational mode of a transfer flow are calculated on the basis of the obtained linear transfer velocities:

$$
\boldsymbol{\omega}_{i}^{n}=\sum_{k} \sum_{l} \frac{\mathbf{r}_{i k l} \times \mathbf{v}_{k l}^{n-1}}{\left|\mathbf{r}_{i k l}\right|^{2}} .
$$

A diagram illustrating the calculation of the angular velocities for the planar case of FCC packing of active elements is presented in Fig. 1(b). Here, not only the 1st, but also more distant coordination spheres are taken into account. This is necessary for the correlation with the case of a simple cubic packing, for which it is impossible to only consider the first sphere when calculating the rotational modes.

Then we calculate the vector $\left(\Delta \boldsymbol{\eta}_{i k}^{n}\right)$ of material flow into the $i$-th element from its neighboring $k$-th element and the corresponding flow vector of mechanical energy into the $i$-th element through the boundary $\left(\Delta \mathbf{A}_{i k}^{n}\right)$ under consideration. 


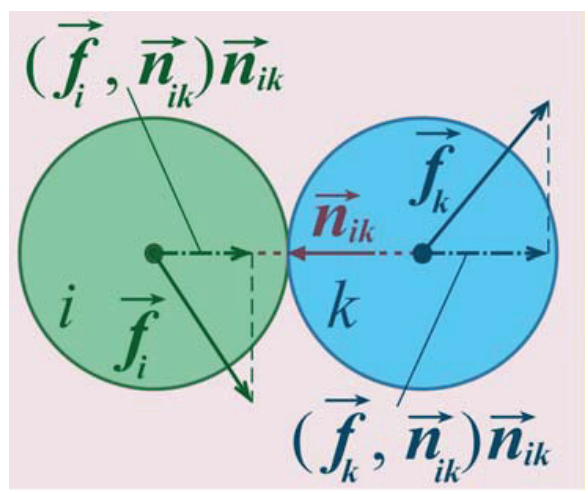

(a)

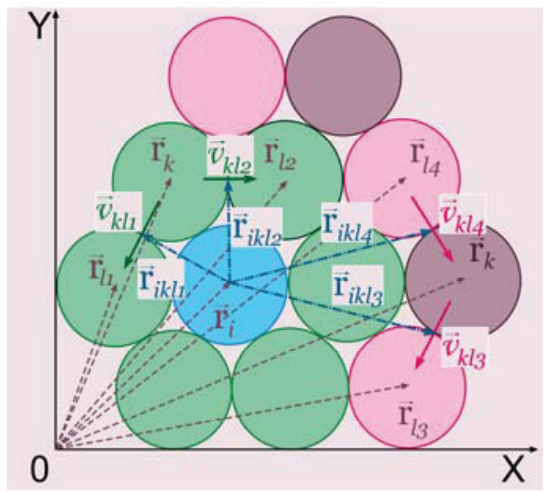

(b)

FIGURE 1. Scheme for calculating the stress at boundaries between elements (a); scheme for calculating the angular velocity of a vortex in the active element of a cellular automaton (b). Two-dimensional case of FCC packing

Hereby we take into account the dissipative term of torsion energy, which leads to the occurrence of defective structures and entropy. This dissipative term is written as follows:

$$
\left[\Delta \mathbf{A}_{d}\right]_{i}^{n}=\frac{k_{\mathrm{tors}} G_{i}\left|\Delta \gamma_{i}^{n}\right| \pi r_{\mathrm{c}}^{3}}{2} \Delta \gamma_{i}^{n}
$$

Here $\Delta \gamma_{i}^{n}$ is an increment of the torsion vector angle of the medium element, $\Delta \gamma_{i}^{n}=\tau \boldsymbol{\omega}_{i}^{n}, G_{i}$ is shear modulus, $k_{\text {tors }}$ is dissipation factor (can be measured experimentally), $r_{\mathrm{c}}$ is the radius of the active element.

\section{NUMERICAL EXPERIMENT}

We used the excitable cellular automaton method to conduct numerical experiments on uniaxial compression of polycrystalline copper specimen perpendicular to the face edges. . Specimens with the size of $100 \times 10 \times 100 \mu \mathrm{m}$ were modeled using a cellular automaton with FCC packing elements; the element size was $1 \mu \mathrm{m}$; the average grain size, $30 \mu \mathrm{m}$. Boundary conditions were set as follows. The front faces were subjected to compressive deformation at $400 \mathrm{~s}^{-1}$; there was no energy exchange through the end faces. The time step was $1 \mathrm{~ns}$. The grain structure of the specimen is shown in Fig. 2(a).

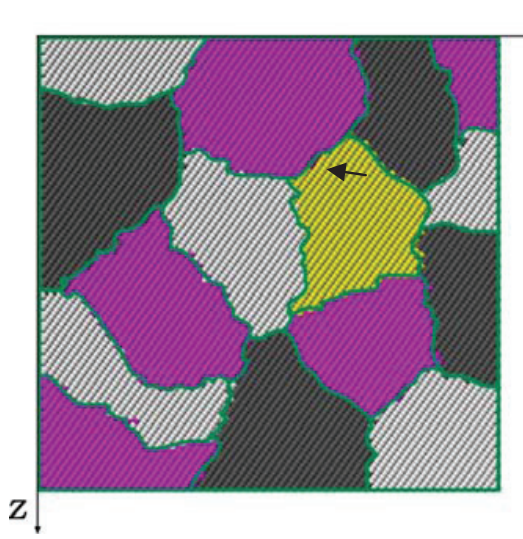

(a)

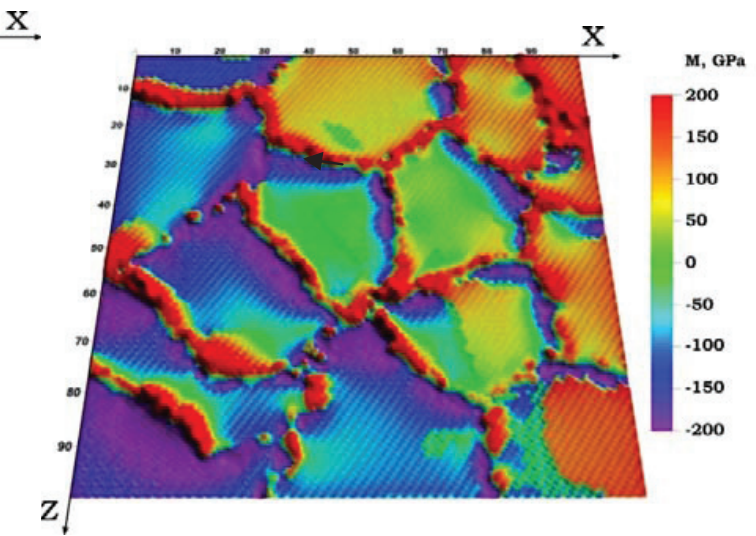

b)

FIGURE 2. Initial grain structure of a specimen under simulation (a) and distribution of values $M=\operatorname{sign}\left(M_{x}+M_{z}\right) \sqrt{M_{x}^{2}+M_{z}^{2}}$ on the front face of the specimen at the time point of 100 microseconds 


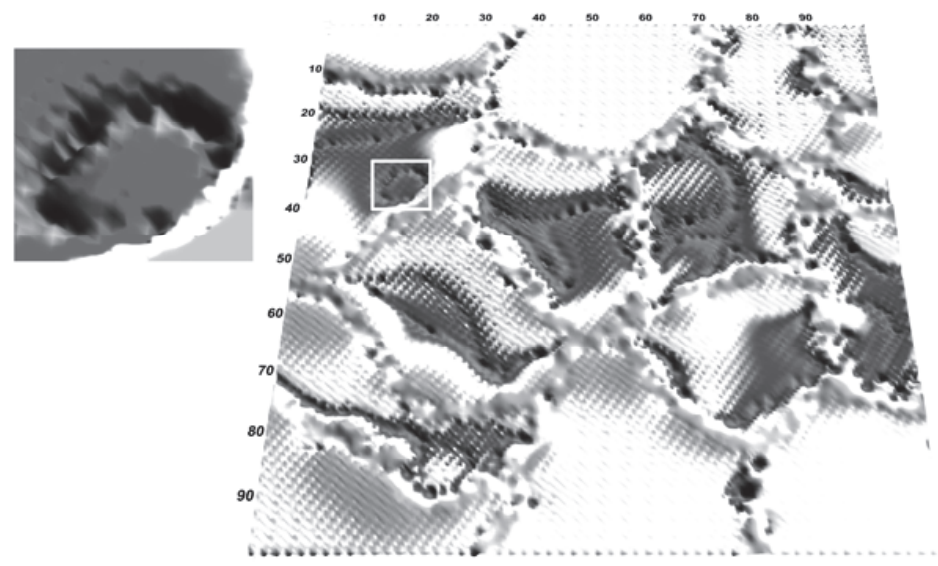

FIGURE 3. Distribution of the common logarithm of $X$-th component of moment of force, $\lg \left|M_{x}\right|$

According to the results of the numerical experiment the distribution of the specific force moment at the front face of the specimen at the time of $100 \mathrm{~ms}$ is given in Fig. 2(b).

We discovered that grain boundaries are sources of the origin of rotational deformation modes. Figure 2(b) shows the distribution of force moment projections onto the plane surface of the specimen. These values of projections correspond to rotational deformation modes along all directions in the plane of the specimen, including grain boundaries.

Since it is at the grain boundaries that the force moment reaches its maximum values, which exceed those in the grain body by several orders, a detailed study of the grain bodies requires a different presentation of the results. Distributions of the logarithm of the $X$-th component of force moment (Fig. 3) were built. The analysis of such distributions shows that some chain rotations fragmenting the grain are formed within the bodies of individual grains. Thus, the chain of rotations can form a closed loop. An example is clearly seen in the selected area of the specimen near the grain triple junction denoted by an arrow in Fig. 2. This effect is explained by the fact that it is energetically impossible to form a large scale curvature in a $3 \mathrm{D}$ crystal, and it is formed by self-organizing vortices at a lower scale level.

\section{CONCLUSION}

The excitable cellular automaton method was supplemented with specific algorithms to calculate the rotational angular velocities of a rotational deformation mode. A dissipative term was introduced into one of the model equations expressing the accumulated elastic energy. The local moments of force are shown to be generated at the grain boundaries.

The analysis of the distribution pattern of rotational deformation modes shows that the grain body fragmentation occurs due to micro rotation sequences being formed in a non-uniform field of local moments of forces within grains.

The simulation is in good agreement with the experiment and confirms the fundamental concepts of physical mesomechanics about the critical role of planar subsystems and rotational wave flows activated therein, which generate defects in the crystal volume.

The study was supported by the projects of the Russian Foundation for Basic Research (Nos. 14-01-00789, 1301-00403 and 13-08-90402) and Grant of the President of the Russian Federation for Support of Leading Scientific Schools (NSh 2817.2014.1).

\section{REFERENCES}

1. V. E. Panin and V. E. Egorushkin, Phys. Mesomech. 14 (5-6), 207 (2011).

2. V. E. Egorushkin, Russ. Phys. J. 35(4), 316 (1992). 